\title{
New technologies-the best is yet to come
}

\author{
Cristiano Pini ${ }^{1,2} \cdot$ Arturo Chiti $^{1,2}$
}

Published online: 15 October 2021

(c) The Author(s), under exclusive licence to Springer-Verlag GmbH Germany, part of Springer Nature 2021

The development of new technologies has always been the most potent driving force of progress in medical sciences, and this is particularly true in the field of medical imaging. The introduction and the rise of techniques such as computed tomography, magnetic resonance imaging and positron emission tomography - to cite a few milestones-were at the core of a technological revolution that radically changed modern medicine and the way we can understand, diagnose and treat diseases.

This moment in time can represent a similar crucial point for the growth of medicine: computer sciences and, in particular, artificial intelligence are on the verge of a leap forward unfathomable just a decade ago; personalized and precision therapies are on the rise and they adopt imaging and molecular imaging techniques at their core; advanced technologies are exponentially getting more precise and more available.

This special issue on new technologies in imaging, edited by Prof. Ali Afshar Oromieh and Prof. Kuangyu Shi from the University of Bern, showcases some of the most exciting and brilliant advancements in the field.

Birnbacher et al.'s review [1] on the state-of-the-art quantitative X-ray gating-based phase-contrast computed tomography showcases the many promising applications of these techniques.

Multi-scale optoacoustic molecular imaging, as thoroughly reviewed by Razansky et al. [2], can provide highresolution real-time data instrumental in understanding

This article is part of the Topical Collection on Technology.

Arturo Chiti

arturo.chiti@hunimed.eu

1 Department of Biomedical Sciences, Humanitas University, Via Rita Levi Montalcini 4Pieve Emanuele, 20090 Milan, Italy

2 Department of Nuclear Medicine, IRCCS Humanitas Research Hospital, via Manzoni 56, 20089 Rozzano, Milan, Italy better the pathophysiology of brain diseases such as Alzheimer's disease, stroke, epilepsy and gliomas.

Li et al.'s review [3] provides a comprehensive insight on the advantages and the challenges of hyperpolarized carbon-13 labelled pyruvate MR. This emerging technology can acquire dynamic metabolic information in a new, promising fashion that could complement limitations posed by established techniques, thus enriching our arsenal.

The introduction of extended field of view PET scanners opened up new possibilities yet to be explored.

Liu et al. [4] compared the kinetic metrics of [18F]FDG between ultra-low activity and full-activity total-body dynamic PET in healthy volunteers, obtaining comparable results and image quality. An essential step towards the application in the clinical setting of ultra-low activity protocols, benefiting from significant reductions in radiation dose and data size.

Alberts et al. [5] evaluated the clinical performance of long field of view PET in a head-to-head intra-individual comparison with a regular scanner. Their findings showcase the excellent results obtainable with total-body PET and the flexibility of the technique, able to provide images of excellent quality, to reduce scan times significantly or to decrease radiation dose, according to the needs and the goals of the examinators.

Magnetic resonance fingerprinting is a promising quantitative MRI framework able to provide fast, high-quality data. Tippareddy et al. review [6] the current state of this new, evolving technology.

Wendler et al. [7] provide a review of molecular imaging techniques applied to precision surgery. Technological advancements in both fields can pave the way for a future of close collaboration between the two communities.

Azargoshasb et al.'s work [8] focused on the production of a novel robotic gamma probe. This new generation device proved to have a relevant impact on surgical dexterity and decision-making, representing a promising step forward to complete integration of radio guidance in precision surgery.

Gaustad et al. [9] developed an intravital microscopy assay using a standard fluorescence microscope. Their assay, 
more affordable than alternative methods, managed to evaluate vascular morphology, tumour growth and response to different treatments.

Khan et al. [10] investigated FDG uptake in an engineered tumour-stroma model with radioluminescence microscopy. Their work demonstrated the technique's feasibility and high-spatial resolution and paved the way for its application on other cancer models, surgical tissues and tumour biopsies.

These works provide a broad scope view of the most promising developments of technology, with a focus on clinical applications, and they all convey one common message: advancements in imaging techniques arguably shaped modern medicine like no other human achievement, and we barely scratched the surface of what can be accomplishedthe best is yet to come.

\section{Declarations}

Informed consent Not applicable.

Ethical approval Institutional Review Board approval was not required because the paper is an Editorial.

Conflict of interest The authors declare no competing interests.

\section{References}

1. Birnbacher L, Braig EM, Pfeiffer D, et al. Quantitative X-ray phase contrast computed tomography with grating interferometry. Eur J Nucl Med Mol Imaging. 2021. https://doi.org/10.1007/ s00259-021-05259-6.
2. Razansky D, Klohs J, Ni R. Multi-scale optoacoustic molecular imaging of brain diseases. Eur J Nucl Med Mol Imaging. 2021. https://doi.org/10.1007/s00259-021-05207-4.

3. Li Y, Vigneron DB, Xu D. Current human brain applications and challenges of dynamic hyperpolarized carbon-13 labeled pyruvate MR metabolic imaging. Eur J Nucl Med Mol Imaging. 2021. https://doi.org/10.1007/s00259-021-05508-8.

4. Liu G, Hu P, Yu H, et al. Ultra-low-activity total-body dynamic PET imaging allows equal performance to full-activity PET imaging for investigating kinetic metrics of $18 \mathrm{~F}-\mathrm{FDG}$ in healthy volunteers. Eur J Nucl Med Mol Imaging. 2021;48:2373-83. https:// doi.org/10.1007/s00259-020-05173-3.

5. Alberts I, Hünermund JN, Prenosil G, et al. Clinical performance of long axial field of view PET/CT: a head-to-head intra-individual comparison of the Biograph Vision Quadra with the Biograph Vision PET/CT. Eur J Nucl Med Mol Imaging. 2021;48:2395404. https://doi.org/10.1007/s00259-021-05282-7.

6. Tippareddy C, Zhao W, Sunshine JL, et al. Magnetic resonance fingerprinting: an overview. Eur J Nucl Med Mol Imaging. 2021. https://doi.org/10.1007/s00259-021-05384-2.

7. Wendler T, van Leeuwen FWB, Navab N, et al. How molecular imaging will enable robotic precision surgery. Eur J Nucl Med Mol Imaging. 2021. https://doi.org/10.1007/s00259-021-05445-6.

8. Azargoshasb S, van Alphen S, Slof LJ, et al. The Click-On gamma probe, a second-generation tethered robotic gamma probe that improves dexterity and surgical decision-making. Eur J Nucl Med Mol Imaging. 2021. https://doi.org/10.1007/s00259-021-05387-z.

9. Gaustad JV, Simonsen TG, Hansem LMK, et al. Intravital microscopy of tumor vessel morphology and function using a standard fluorescence microscope. Eur J Nucl Med Mol Imaging. 2021;48:3089-100. https://doi.org/10.1007/s00259-021-05243-0.

10. Khan S, Kim S, Yang YP, et al. High-resolution radioluminescence microscopy of FDG uptake in an engineered 3D tumorstoma model. Eur J Nucl Med Mol Imaging. 2021;48:3400-7. https://doi.org/10.1007/s00259-021-05364-6.

Publisher's note Springer Nature remains neutral with regard to jurisdictional claims in published maps and institutional affiliations. 